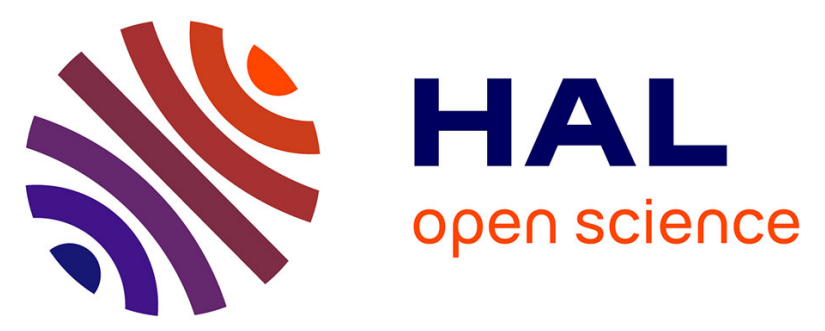

\title{
Localized probabilistic and dominating set based algorithm for efficient information dissemination in ad hoc networks
}

\author{
Michaël Hauspie, Amandine Panier, David Simplot-Ryl
}

\section{To cite this version:}

Michaël Hauspie, Amandine Panier, David Simplot-Ryl. Localized probabilistic and dominating set based algorithm for efficient information dissemination in ad hoc networks. Mobile Ad-Hoc and Sensors Systems, 2004, Fort Lauderdale, United States. pp.11-20. hal-00616855

\section{HAL Id: hal-00616855 \\ https://hal.science/hal-00616855}

Submitted on 24 Aug 2011

HAL is a multi-disciplinary open access archive for the deposit and dissemination of scientific research documents, whether they are published or not. The documents may come from teaching and research institutions in France or abroad, or from public or private research centers.
L'archive ouverte pluridisciplinaire HAL, est destinée au dépôt et à la diffusion de documents scientifiques de niveau recherche, publiés ou non, émanant des établissements d'enseignement et de recherche français ou étrangers, des laboratoires publics ou privés. 


\title{
Localized probabilistic and dominating set based algorithm for efficient information dissemination in ad hoc networks
}

\author{
Michaël Hauspie Amandine Panier \\ David Simplot-Ryl \\ IRCICA/LIFL, Univ. Lille 1, UMR CNRS 8022 \\ INRIA Futurs, POPS research group \\ Bât. M3, Cité Scientifique \\ 59655 Villeneuve d'Ascq Cedex, France \\ email: \{hauspie,panier, simplot\}@lifl.fr
}

\begin{abstract}
Ad hoc networks are autonomous dynamic networks composed of mobile devices like personal digital assistants (PDA) for instance. In such mobile networks, lack of infrastructure leads to non trivial information discovery and dissemination. A scheme in which a unique object centralizes information is not efficient for many reasons. In this paper, we propose a probabilistic algorithm to satisfactorily distribute an information token among nodes forming the network by using localized datas. Then, in order to limit the number of memorizing nodes, we propose to make memorize nodes belonging to a dominating set.
\end{abstract}

\section{Introduction}

Ad hoc networks are autonomous dynamic networks composed of mobile devices like personal digital assistants (PDA) for instance. In such mobile networks, lack of infrastructure leads to non trivial information discovery and dissemination. A scheme in which a unique object centralizes information is not efficient for many reasons. For instance, let us consider a location server which provides geographic location of nodes. Such server can be used in several layers: in geographical routing [9] or context-aware applications.

Indeed, it is difficult to maintain a centralized structure because of network topology variation. Firstly, if we do not suppose any fixed access point, maintaining the server location is expensive. Secondly, even if we have a fixed access point, nodes which are far from the server are penalized since request time increases with distance. Thirdly, this kind of architecture raises an issue: the server may become the seat of a bottleneck. As a matter of fact, all requests are sent to the single server. This issue is known in wire infrastructures. This is why research focuses on proposing distributed versions of known centralized algorithms in the wired world. Finally, and it is again a well known issue in wired infrastructures, if the server falls down, the entire network is paralyzed. The ideal dissemination would be each node knows all available information in the network but considering that mobiles are limited in memory space and energy, it is not a realistic solution.

In this paper, we propose a probabilistic algorithm to satisfactorily distribute an information token among nodes forming the network, that is to say only a given number of nodes will memorize. Then, in order to limit the number of memorizing nodes, we propose to make memorize nodes belonging to a dominating set. A dominating node decides to memorize according to a probability. In our proposal, a node considers as priority both its id and the id of the source of information. We do not consider a particular type of information and we discuss on how efficiently realize the dissemination. This kind of dissemination would find application in service discovery where mobiles may need to access services or in routing for example.

The paper is organized as follows. In the next section we will review some architectural supports which have been set up in this two particular fields of application and we will deal with dominating sets. In Section 3, we will expose our proposal. 
Then, in Section 4, we will analyze the results of the experiments. Finally, in Section 5, we will sum up our proposal and give details for future work.

\section{Literature Review}

In this section, we first deal with some architectural supports proposed in the literature for service discovery and for geographical routing. Then, we deal with dominating sets.

\subsection{Architectural Supports for Service Discovery and Geographical Routing}

Information dissemination is something important for service discovery. An efficient information dissemination can allow to reduce message overhead and request time.

In the literature, proposed service discovery architectures are generally centralized although it is not a suitable solution for ad hoc networks. In [6], the authors try to show that it is not inevitably a bad choice. They deal with Directory Agents hosted by nodes forming a dominating set [4]. This DAs allow servers to record services they provide. The disadvantage of this solution is that the memorizing nodes are always the same. The nodes forming the "virtual backbone" are penalized.

A performance evaluation of different Postquery strategies is proposed in [8]. A Post-query strategy executes Post-query protocols (servers publish services they provide according to the Posting protocol and clients request for services according to the Querying protocol) in rounds. Five Post-query strategies are confronted : the greedy strategy, the incremental strategy, the uniform memoryless strategy, the with memory strategy and the conservative strategy. The greedy strategy consists in making all nodes publish to or query all nodes in the network; the incremental strategy consists in making the set of nodes to which other nodes publish and the set of nodes other nodes query bigger each round; the uniform memoryless strategy consists in making all nodes publish to or query a random set of nodes; the with memory strategy consists in making all nodes publish to or query each round nodes not yet contacted; the conservative strategy consists in making all nodes publish to or query its one-hop neighbors. The greedy strategy is obviously unsuitable since nodes are limited in memory space. The uniform memoryless strategy may be suitable if the set of nodes is small and the way memorizing nodes are distributed is interesting, but the dissemination of information does not follow any reproducible property. The with memory strategy tends to make all nodes knowing about all available information in the network. The conservative strategy, implies a non satisfactory dissemination of information since nodes wishing to get the information token provided by the source will reduce the cost of the request by one hop in the best case.

In [5], Karlsson et al. evaluate heuristics for replica placement in wide-area systems and propose a methodology for heuristic selection. They introduce constraints such as a threshold for the average latency for example. Thus, they obtain a system of constraints. The designer has to calculate the general lower bound and the lower bounds for all classes of heuristics that could be suitable for the system. The most suitable heuristic is the one with the lowest bound. If this lower bound is close to the general lower bound, there exists no heuristic among non tested classes of heuristics that would really be more suitable than the heuristic with this lower bound. This approach would obviously not be suitable for mobile networks since it is an off-line approach, where the designer has to configure the parameters and to choose an appropriate heuristic each time the topologie changes.

In the same way as for service discovery, information dissemination is important for geographical routing since mobiles need to know the location of the destination they want to reach. To access such an information token, the sender node can broadcast a request for location and wait for a reply for example. Each node knows its location thanks to a positioning system such as GPS [1].

Grid Location Service [7] is a distributed service location. Each node updates its location to location servers and is able to be a location server as well. As we move away from a node, its location servers are scattered more and more; this is possible since the network is divided into bigger and bigger squares. A level-0 square corresponds to the unit square. A level- $n$ square is composed of four level- $(n-1)$ squares. Each node has to choose one node as location server in each of the three squares taking part to the formation of a higher level-square with it. Figure 1 shows in which squares node B will have to choose its location servers. Since the number of location servers for a node decreases as we move away from it, the total number of a node location servers tends to be minimized. A node updates its location to its 
location servers when it has moved a given distance $d$ away from its last updated location. The main disadvantage of this proposal is that it implies inevitably the use of GPS; this is not suitable for information dissemination in the general case. Moreover, it may happen that a node goes far away to find a data instead of finding it near to it, increasing the overhead and the query delay; this is the case for border nodes within a square since the distance between a border node wishing to reach another border node standing next to him in a different square and a location server of this node, is ineluctably longer than the distance between these two nodes.

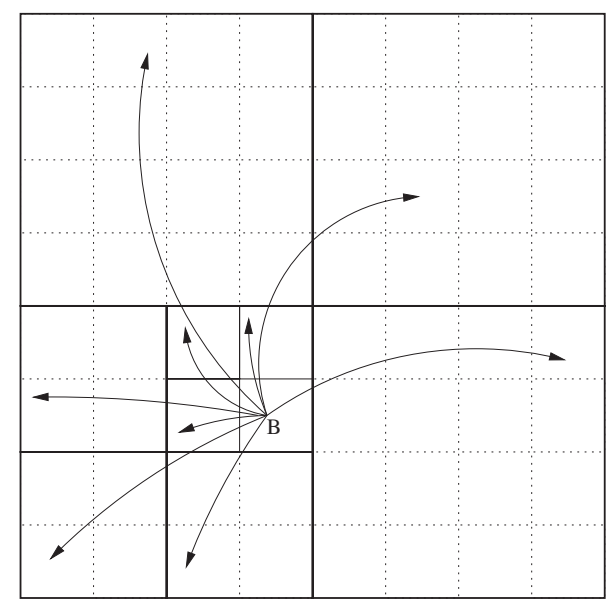

Figure 1. Example of Grid distribution for node B location.

In Tribe [10], a node updates its location only to one another node which is discovered on demand: its anchor. Each node in the network has a universal identifier, a virtual identifier, a relative address and a control area. A node calculates its virtual identifier in the addressing space from its universal identifier. Its anchor is then the node whom control area contains its virtual address. The anchor knows the relative address of the node and its current location. When a node needs to communicate with another node, it first has to request for the current location of the destination. To do so, the node has to calculate, in the same way as the destination node, the virtual address to find the node knowing the desired location. This is possible since the universal addresses and the conversion function in respective virtual addresses are supposed to be known by each node forming the network. When a node has to forward a packet, it chooses in its neighborhood the node whom control area is closed to the control area containing the identifier of the destination. Contrary to Grid, Tribe does not use geographical information to determine the nodes which will memorize the location of other nodes and to route the packets. The disadvantage is that the anchor of a node may be faraway from the node thus increasing message overhead and request time.

\subsection{Dominating Sets}

In this section, we present dominating sets and the algorithm we will use in our proposal to construct them.

Dominating sets can be used in routing [4] to determine nodes belonging to the search space for routes and in broadcasting [11] to determine forwarding nodes. A set of nodes is dominating if all nodes belong to this set or have a neighbor belonging to this set. A dominating set is a connected dominating set (CDS) if all nodes in the set can communicate with all the other nodes in the set by using multihop links. When a node decides to broadcast a message, if nodes belonging to the CDS forward it, all the network is covered. The interest is to minimize the dominating set size so as to limit the number of forwarding nodes.

$\mathrm{Wu}$ and $\mathrm{Li}$ propose a marking algorithm providing a CDS in [12]. The first step of the algorithm consists in marking nodes that possess at least two neighbors that are not directly connected. Then, two rules are successively used to reduce the size of the CDS previously obtained. The first rule (Rule 1) consists in unmarking a node if all of its neighbors are also neighbors of another marked node with higher priority than it. The second rule (Rule 2) consists in unmarking a node if all of its neighbors are also neighbors of two other directly connected marked nodes with higher priorities than it. In [4], Dai et al. introduce a new rule, called Rule $k$, to minimize the size of the dominating set obtained. This rule consists in unmarking a node if its neighborhood is "covered" by a connected set of nodes with higher priorities than it. It is better than Rule 1 and Rule 2 in reducing the number of dominating nodes.

In [2], the authors propose a rule to determine if a node is not a dominating node. This proposal is a variation of Rule $k$. A node $u$ is not dominating if the set of its neighbors with higher priorities than it is connected and "covers" all its neighbors. This is the definition we use in our proposal.

In algorithms aiming to find dominating sets, this is generally the node id which is used as prior- 
ity. The disadvantage is that the penalized nodes are always the same. It is not suitable for ad hoc networks since mobiles are limited in memory space.

All reviewed proposals in Section 2.1 do not take care about available memory space of objects acting as servers of information. Moreover, the designed architectures may be difficult to maintain due to the mobility of the network. We propose a localized probabilistic algorithm to efficiently disseminate information across the network. We further improve this algorithm using dominating sets.

\section{Probabilistic dissemination}

In this section, we will focus on describing the property hidden behind the idea of an efficient information dissemination and how we achieve to verify this property using only local data.

\subsection{Efficient information dissemination}

We believe that an efficient dissemination should balance the number of nodes needed to cache information and the cost induced by requesting it. We also believe that a node looking for an information token should find it or a reference to it on a node closer to the source than itself. On one hand, the closer is the node that caches the token from the node that makes the request, the lower will be the cost of the research. On the other hand, it grows up the number of nodes that are needed to disseminate the token and thus, the cache size needed if more than one token is disseminated.

We can formalize this idea by the following definition. We consider a network modeled as a unit disk graph [3] $G(V, E)$ where $V$ is the set of nodes and $E$ is the set of edges. An edge exists between two nodes if they can communicate through one direct wireless link (i.e. they are within each other's radio radius).

Definition 1 Let $u$ be a node that has an information token $I_{u}$ to disseminate across the network. Let $v$ be a node and let Cache $(v)$ be the set of tokens that are stored in the cache of node $v . I_{u}$ is said well disseminated if the following property is verified :

$$
\forall v \in V, m<d(u, v) \leq M,\left\{\begin{array}{l}
I_{u} \in \operatorname{Cache}(v) \\
\text { or } \\
\exists w \in V \text { s.t. } \\
I_{u} \in \operatorname{Cache}(w), \\
d(v, w)<\lambda d(v, u), \\
d(u, w)<d(u, v),
\end{array}\right.
$$

where $m$ and $M$ are the minimum and the maximum distance between which we consider the criterion, $d(x, y)$ is the distance between node $x$ and node $y$ in hops and $\lambda$ is a distance factor $(\lambda \in] 0,1[)$.

The parameter $\lambda$ in the above definition is the factor tweaking the balance between memory size and research cost. The lower it is, the lower is the cost of research but the higher is the number of nodes needed to cache the token. For instance, a fair balance would be achieved with $\lambda=\frac{1}{2}$.

\subsection{Perfect case}

Considering a perfect case, where we can place nodes wherever we want, we can evaluate where to place memorizing nodes to verify the property using as few nodes as possible. The main idea of our algorithm will then be to deduce a probabilistic law for selecting the nodes that will cache the token in order to average the perfect locations as accurately as possible. So, the first thing to do is to find the perfect locations of the nodes that will cache the token. In that perfect but theoretical case, we assimilate hop-distance to euclidean distance. In the real case, we will run the algorithm using hop-distance because we do not have any positionning system. As we will shown after, using hop-distance is still enough to verify the property.

As we consider the criterion starting at distance $m$, the first nodes that need to cache the token will be located at a distance $m$ to the source. Those nodes are at the first rank, called $r_{0}$ in the following. Each of those nodes will cover a zone located at a distance $\lambda m$ around them. A zone is said covered if all nodes located inside it satisfy the property given in Definition 1. Thus, we need to distribute a number $a_{0}$ of nodes on the circle of center the source node and radius $m$. From here, we can give the rule for constructing rank $r_{n+1}$ from rank $r_{n}$ (which will increase the covered area). The construction of rank $r_{1}$ from rank $r_{0}$ is depicted in Figure 2. Rank $r_{n+1}$ is constructed from rank 
$r_{n}$ by placing a node on the intersection of each couple of circles of centers each successive pair of nodes of rank $r_{n}$ and radius $\lambda m_{n}$ where $m_{n}$ is the distance between the nodes of rank $r_{n}$ and the source. A full view for ranks $r_{0}$ and $r_{1}$ is given in Figure 3.a and 3.b.

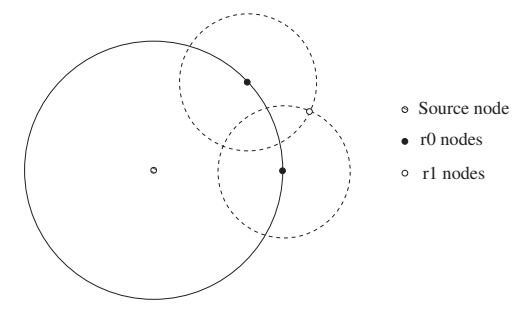

Figure 2. Constructing $r_{1}$ from $r_{0}$.

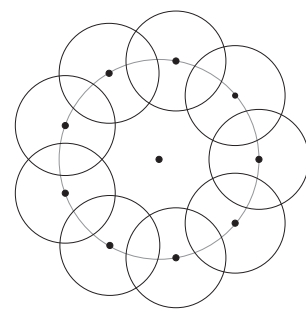

(a) $r_{0}$

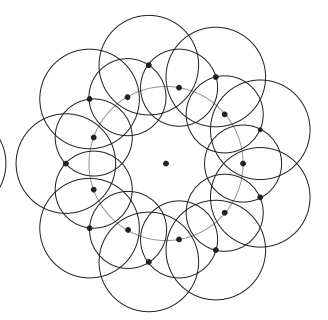

(b) $r_{0}$ and $r_{1}$

\section{Figure 3. Area covered by ranks $r_{0}$ and} $r_{1}$.

This construction algorithm introduces a condition on the maximal distance between nodes of a same rank. Nodes of rank $r_{n}$ must be at most at distance $2 \lambda m_{n}$ to each other to ensure that the circles intersect. We can then define a condition on the minimum number of nodes to place on rank $r_{0}$ :

$$
a_{0}>a_{\min }=\frac{2 \pi}{\arccos \left(1-2 \lambda^{2}\right)} .
$$

To satisfy the condition, we consider using $a_{0}=$ $a_{\text {min }}+k, k \in \mathbb{N}^{*}$. We need to find the best value of $k$ so that the whole desired area is covered by a minimum number of nodes. This can be easily done by computing it for some values of $k$. Figure 4 shows the number of nodes needed to cover an area going from 2 hops to the source to 20 hops with $\lambda=\frac{1}{2}$. In that case, we can see that the good values of $k$ are 3 or 4 .

In our construction, each rank has the same number of nodes, that is $a_{0}=a_{1}=\cdots=a_{n}$. Thus, we are able to know for each rank, how many nodes should cache the token so that the full

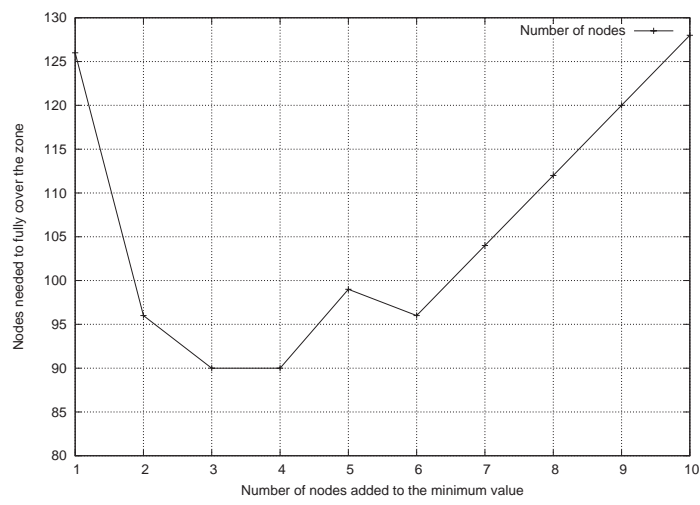

Figure 4. Number of nodes to add to $a_{\min }$ to cover all the zone.

area is covered (i.e. each node in the area satisfies the property given in Definition 1). The last thing needed is to know, for each rank, its distance to the source. Again from simple geometrical properties, we can deduce $m_{n}$ (the distance of rank $r_{n}$ to the source):

$$
m_{n}=m_{0} \cdot \tau^{n}
$$

where $\tau$ is given by

$$
\begin{array}{r}
\tau=\sqrt{1-\frac{1}{2}\left(1-\cos \frac{2 \pi}{a_{0}}\right)}+ \\
\sqrt{\lambda^{2}-\frac{1}{2}\left(1-\cos \frac{2 \pi}{a_{0}}\right)} .
\end{array}
$$

Given the equations (1) and (2), we know where to ideally place nodes that will cache the token so as to verify the property given in Definition 1 . It is then easy to define a probabilistic rule $P(d)$ so that a node $u$ located at a distance $d$ to the source $S$ can decide by itself either it should cache the token or not :

$P(d)= \begin{cases}\frac{a_{0}}{N C_{d}} a_{0} & i \text { s.t. } m_{i}=d, \\ \overline{N C_{d}+N C_{d+1}} & \text { s.t. } m_{i} \notin \mathbb{N}, \\ & \text { and }\left\lfloor m_{i}\right\rfloor=d(S, u), \\ 0 & \text { otherwise, }\end{cases}$

where $N C_{d}$ is the number of nodes located at distance $d$ to the source. $N C_{d}$ can be approximated simply by using the surface of the ring of outer radius $R m_{i}$ and inner radius $R(d-1)$. This surface 
is given by

$$
S=\pi R^{2} d^{2}-\pi R^{2}(d-1)^{2}=\pi R^{2}(2 d-1)
$$

where $R$ is the transmission radius. Thus, $N C_{d}$ can be approximated by the following formula

$$
N C_{d}=D(2 d-1)
$$

where $D$ is the number of nodes per communication zone (i.e. the density of the network). The accuracy of this formula is shown in Figure 6.

Thus, the publication algorithm to run is given in Figure 5.

$$
\begin{aligned}
& \text { foreach node } u \in G(V, E) \\
& \quad p:=\operatorname{rand}() ; \\
& \quad \underline{\text { if } p<P(u)} \\
& \quad \text { Cache the token in the node } u \\
& \quad \underline{\mathbf{f}} \\
& \text { end }
\end{aligned}
$$

\section{Figure 5. Information publication al- gorithm.}

\subsection{Localized protocol}

As the global density can be estimated by the local density, each node has the required knowledge to execute the algorithm given in Figure 5 in a localized manner. The publish is done using a broadcast protocol. Each node receiving the publication packet generates a random number and checks against its probability. The distance to the source is given by the publication packet.

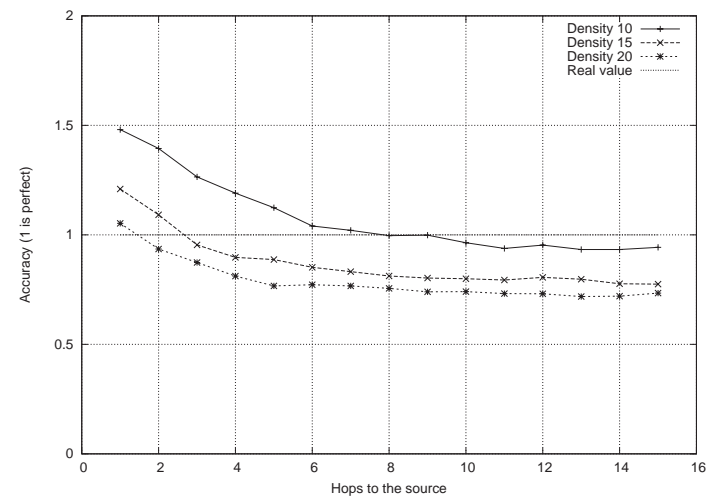

Figure 6. $N C_{d}$ evaluation accuracy.

The main advantage of the algorithm is that publication is done using only one broadcast. If a node needs to look for a token, it broadcasts a request in a small area around him. A node that receives this request and that has cached the token can reply by providing the information token or sending the address of the token publisher. If no node receives the request, another broadcast is done with a bigger area. The process is iterated until a node that knows something about the requested token replies. As information is well disseminated, the request process will not iterate much and the cost of research will remain low.

The next section exposes how this proposal can be enhanced through the use of dominating sets.

\subsection{Using dominating sets}

Although our protocol works well, there is still a problem induced by this fully probabilistic algorithm. As the nodes take the decision by themselves without being aware of their neighborhood, more than one node may store the information token where only one would be enough. If a node only considers probability to memorize at each rank, it may happen that two nodes close to each other memorize the same token. We believe that the use of dominating sets should lower the number of nodes storing and thus the memory use induced by the dissemination.

Indeed, the property can still be verified if only dominating nodes store the token. The goal is then to run the algorithm given in Figure 5 no more on all nodes but only on dominating ones.

\subsubsection{Construction of Dominating Sets}

If we construct only one dominating set using the nodes id as priority, we would always use the same nodes. That is not acceptable as their cache is not unlimited. Thus, we construct several dominating sets using the node id and the source id as priority. Then, the set will be different for each source, distributing the tokens among the nodes with a fair balance, optimizing the cache size needed in each node. Moreover, we only use a non connected dominating set because it would raise the number of potential nodes without enhancing our proposal. Thus, the rule for determining if a node $u$ is not dominating is changed to $u$ is not dominating if the set of its neighbors with higher priorities than it "covers" all its neighbors. 


\subsubsection{Memorization algorithm}

Each node knows for each source if it belongs to a dominating set. Only nodes belonging to a dominating set for a given source check against the probability to memorize the token provided by this source. Thus, the property is still verified and the number of memorizing nodes is reduced.

The probability for a node to memorize given by (3) is modified since $N C_{d}$ is now the number of dominating nodes which can be approximated by

$$
N C_{d}=\pi d,
$$

as shown in figure 7. This formula comes from the fact that if dominating nodes were placed on a circle of radius $d R$, the distance between them would be $2 R$ (where $R$ is the communication radius). Although the formula overestimates the number of dominating nodes, it only occurs at a relatively far distance to the source. Thus, the results on the probability still fit our needs (see Section 4.2).

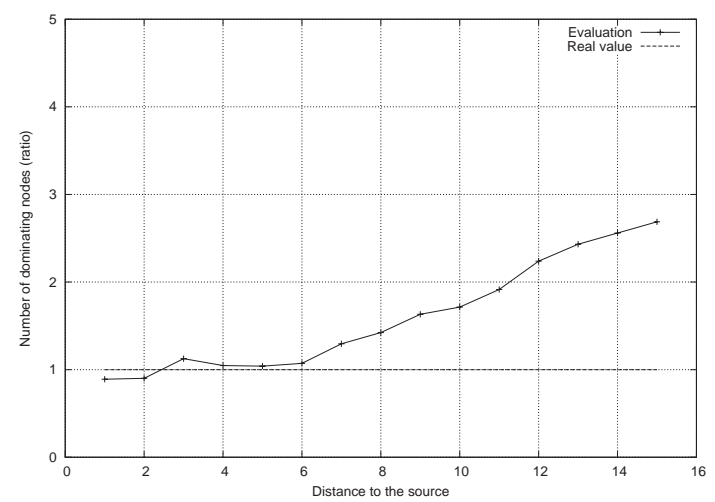

Figure 7. $N C_{d}$ evaluation accuracy in the case of dominating set

\section{Experiments}

\subsection{Without dominating sets}

In order to evaluate our protocol, we run experiments on a 500 nodes unit graph. Nodes are distributed uniformly in a square area. The communication range is 10 meters. A node is selected at the center of the area to be the source of the information token. It broadcasts a publication packet that includes its identifier and some data about the token it wishes to publish. These data depend on the type of information that is published. For instance, in the case of service publication, it can include the service description which will be stored with the id of the publisher. Nodes forwarding the broadcast packet run the algorithm given in Figure 5 to decide either they should store the token or not. First, we present the results obtained with $\lambda=\frac{1}{2}$ as it is representative of a fair balance between cache size and research cost. Then, we will show how we can modify the cache needs through the change of the value of $\lambda$.

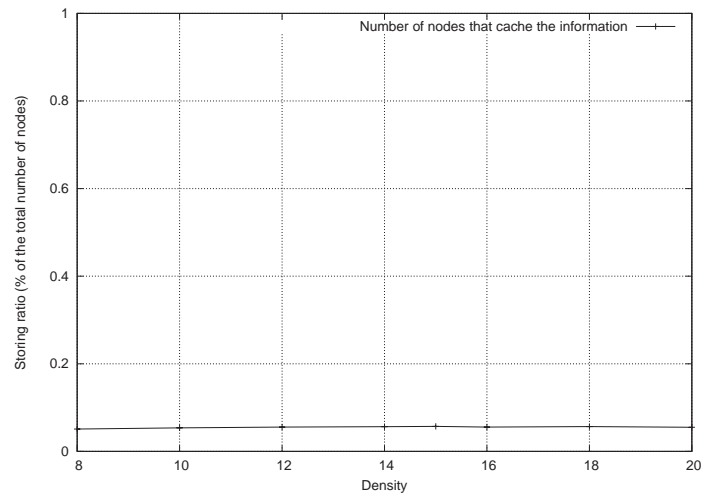

(a) Number of nodes caching the information token

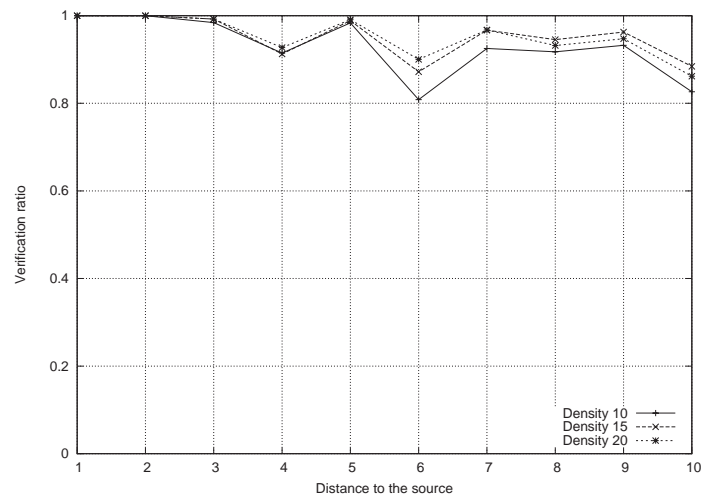

(b) Number of nodes that verify the property after publication

Figure 8. Efficiency of the publication algorithm.

Figures 8.a and 8.b, show the proportion of nodes that are storing the token and how the property given in Definition 1 is verified across the network. We can see that we achieve to strongly verify the property around the source using only a small amount of nodes. With this results, we can expect to be able to manage much more sources and verify the property for all these sources without leading to huge cache size needs.

Figure 9 shows that our expectation is verified 


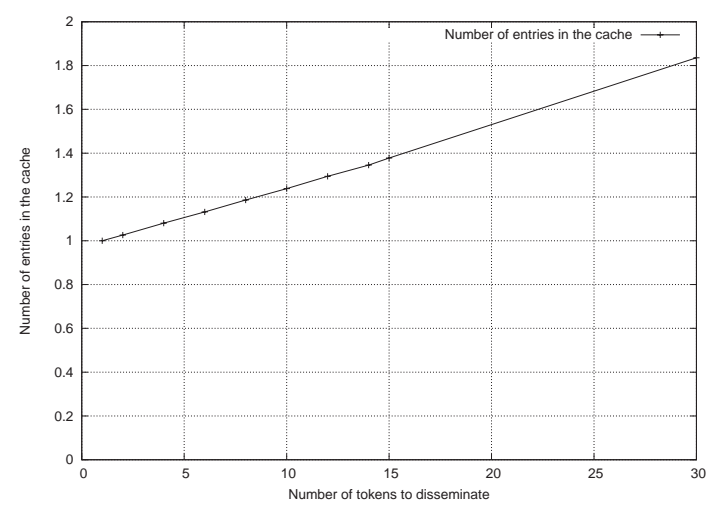

Figure 9. Average number of entries in the cache of nodes that are storing.

as the number of entries in the cache of the nodes remains very low while increasing the number of nodes.

The influence of $\lambda$ for the publication is shown in Figure 10. The values are given for density 15 and $\lambda=0.3,0.7$ and 0.9 . We can see that, as we expected, modifying $\lambda$ can change the average cache size. Moreover, the property is still verified in each case.

\subsection{Enhancement through dominating sets use}

As we can see in Figure 11.b, we achieved to lower the number of nodes needed to verify the property by using non connected dominating sets. Moreover, Figure 11.a shows that the property is still verified which was our goal. Figure 11.c shows that the priority used to construct the dominating set (a hash between source id and node id) well balance the selected nodes when using multiple sources. Indeed, the cache size in the nodes that are storing tokens is lower than the one when only the probabilistic algorithm is used.

\section{Conclusion}

In this paper, we proposed a probabilistic method for performing an efficient information dissemination in a mobile ad hoc network. We first proposed a criterion of efficient information dissemination which depends on the balance of request and memory cost we want to address. We shown through experimental results that our proposal fits our objective which was to verify our criterion using little memory and a simple algorithm that can run on very small nodes. Then, we improved this proposal by using dominating sets and shown through experimental results that the property is still verified.

Our experiments shown that our algorithm should be suitable for a large population which disseminates a large amount of information across the network. For this purpose, we must work on an efficient cache policy to use when no more memory is available on small objects.

Our next work is to evaluate the cost of the request to verify that our protocol actually optimizes it.

\section{References}

[1] The Global Positioning System FAQ, July 1997. URL: http://www.gpsy.com/gpsinfo/gps-faq.txt.

[2] J. Carle and D. Simplot-Ryl. Energy efficient area monitoring by sensor networks. IEEE Computer Magazine, (37):40-46, 2004.

[3] B. N. Clark, C. J. Colbourn, and D. S. Johnson. Unit disk graphs. Discrete Mathematics, 86:165177, 1990.

[4] F. Dai and J. Wu. Distributed dominant pruning in ad hoc networks. In Proc. of IEEE International Conf. on Communications (ICC'03), May 2003.

[5] M. Karlsson and C. Karamanolis. Choosing replica placement heuristics for wide-area systems. In Proc. of the 24th International Conference on Distributed Computing Systems (ICDCS'04), 2004.

[6] U. C. Kozat and L. Tassiulas. Service discovery in mobile ad hoc networks: an overall perspective on architectural choices and network layer support issues. Adhoc Networks, 2:23-44, 2004.

[7] J. Li. A scalable location service for geographic ad hoc routing. Master's thesis, Massachusetts Institute of Technology, January 2001.

[8] H. Luo and M. Barbeau. Performance evaluation of service discovery strategies in ad hoc networks. In 2nd Annual Conference on Communication Networks and Services Research (CNSR 2004), Canada, may 2004.

[9] C. Perkins and P. Bhagwat. Highly dynamic destination-sequenced distance vector (DSDV) for mobile computers. ACM SIGCOMM '94 Computer Communications Review, 24(4):234-244, Oct. 1994.

[10] A. C. Viana, M. D. de Amorim, S. Fdida, and J. F. de Rezende. Indirect routing using distributed location information. In Proc. of IEEE International Conference on Pervasive Computing and Communications (PerCom'03), Dallas-Fort Worth, Texas, March 2003.

[11] J. Wu and F. Dai. Broadcasting in ad hoc networks based on self-pruning. In Proc. of the 22nd 


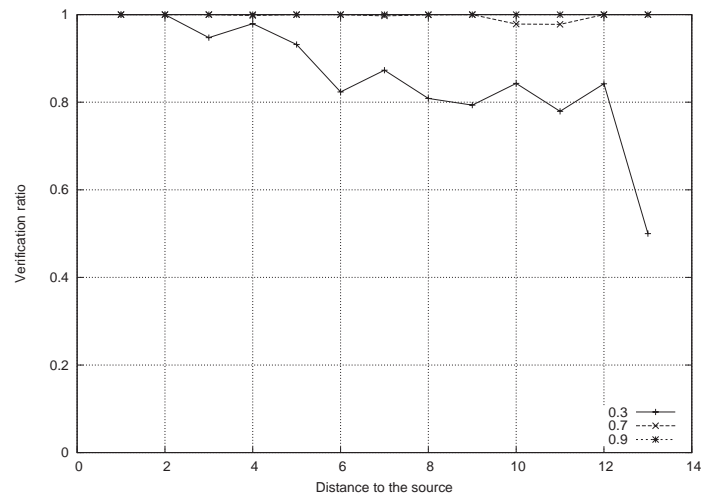

(a) Influence on property verification

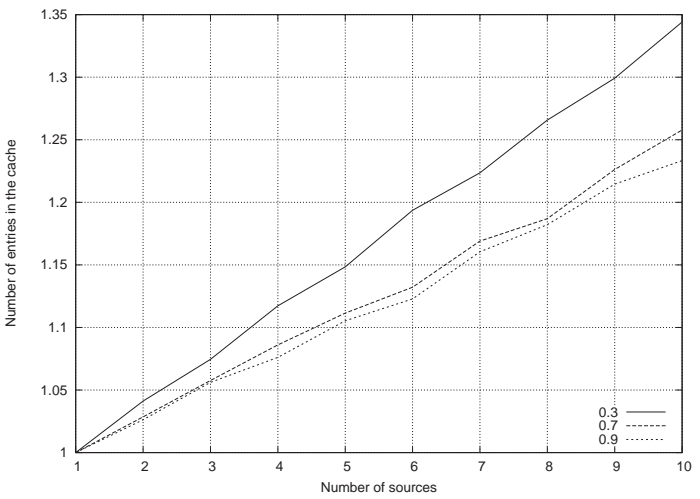

(b) Influence on cache size

Figure 10. Influence of $\lambda$.

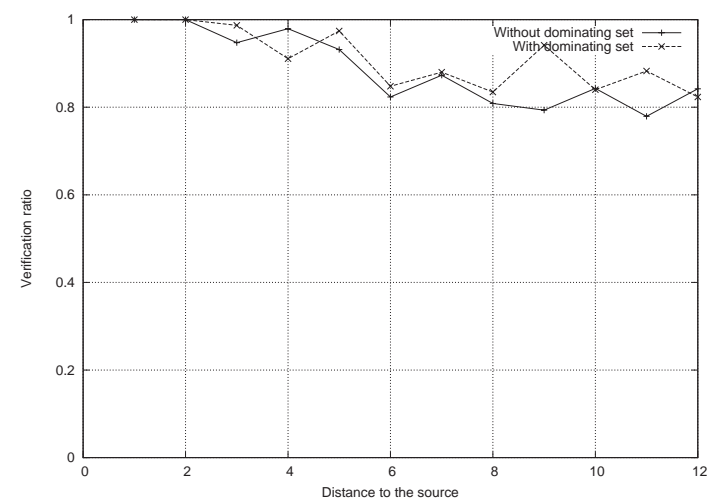

(a) Influence on property verivication

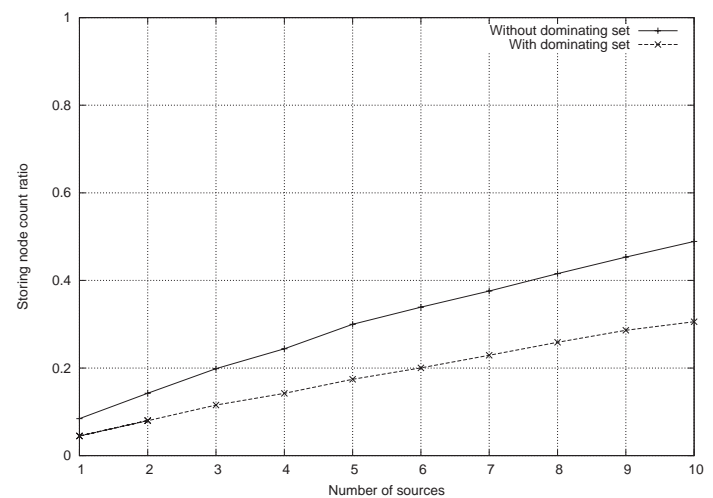

(b) Influence on number of nodes used

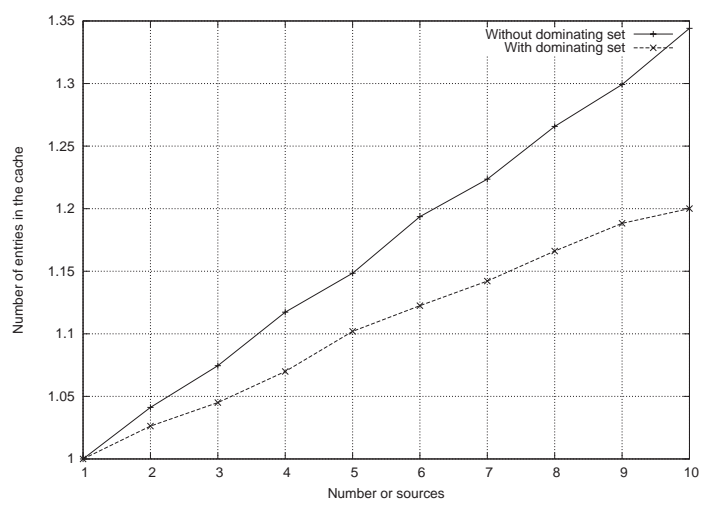

(c) Influence on cache size for storing nodes

Figure 11. Enhancement provided by the use of dominating sets. 
Annual Joint Conference of the IEEE Computer and Communications Societies (INFOCOM), San Francisco, 2003.

[12] J. Wu and H. Li. On calculating connected dominating set for efficient routing in ad hoc wireless networks. In Proc. of the Third International Workshop on Discrete Algorithms and Methods for Mobile Computing and Communications (DiaLM), pages 7-14, 1999. 